\title{
Table of legislation
}

\section{European}

Charter of Fundamental Rights

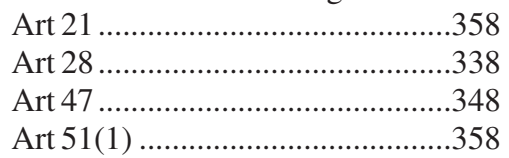

Commission Banking Communication (2013): Communication from the Commission on the application, from 1 August 2013, of State aid rules to support measures in favour of banks in the context of the financial crisis (2013/C 216/01) 268

Commission Best Practice Guidelines 2013: The Commission's Model Texts for Divestiture Commitments and the Trustee Mandate under the EC Merger Regulation, 5.12.2013 .... 262, 266

Commission Guidelines on the application of Art 81(3) [now Art 101(3) TFEU] of the Treaty [2004] OJ C 101/97 340

Commission, Guidelines on Horizontal Co-Operation Agreements, (2011) OJ C11 $140,153,157,158$

Commission Notice, Guidelines on Vertical Restraints, OJ C 130, 19.05.2010, p. 1 339,340

Commission Notice on best practices for the conduct of proceedings concerning Articles 101 and 102 TFEU, OJ C 308, 20.10.2011, pp. 6-32 267

Commission Notice on Immunity from fines and reduction of fines in cartel cases, OJ C 298, 8.12.2006, p. 1 , as amended 327
Commission Remedies Notice 2001: Commission Notice on remedies acceptable under Council Regulation (EEC) No 4064/89 and under Commission Regulation (EC) No 447/98, OJ C 68, 02.03.2001, p. 3 262,266

Commission Remedies Notice: Commission notice on remedies acceptable under Council Regulation (EC) No 139/2004 and under Commission Regulation (EC) No 802/2004, OJ C 267, 22.10.2008, pp. 1-27 .... 262, 267, $273,275,278,279$

Directive 77/249/EEC lawyers freedom to provide services (1977) OJ L78/17 143,150

Directive 84/450/EEC Misleading Advertising [1984] OJ L 250/ 17

Directive 85/374/EEC Product

Liability, OJ L 210, 7.8.1985, p. 29

Art 6(1) 355

Directive 89/106/EEC Construction products [1988] OJ L40/12

Art 7/2. 159

Directive 93/13/EEC Unfair terms in consumer contracts, OJ L 95, 21.04.1993 p. 29 ........ 4, 338, 341

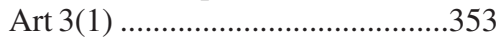
Art 4(2) .........................................354 Directive 93/104/EC Working Time (OJ 1993 L 307, p. 18 ................... 359

Directive 95/46/EC Data Protection [1995] OJ L 281/31 .... 43, 44, 263 rec 49 ..........................................263 Art 18(2) .......................................269 
Directive 98/5/EC practice of the profession of lawyer on a permanent basis in a Member State other than that in which the qualification was obtained [1998] OJ L77/36 143,150

Directive 1999/44/EC Consumer Sales [1991] OJ L 171/12

Art 2(1) .357

Art 2(2)(d)..... .68

Directive 2000/31/EC E-commerce ... 4 Directive 2001/95/EC General Product Safety [2002] OJ L11/4 150 ,

Art 3/3 355 150

Directive 2003/6/EC Insider dealing and market manipulation (market abuse) [2003] OJ L96/16

Art 11 150

Art 12 150

Directive 2004/18/EC Public works contracts, public supply contracts and public service contracts, OJ L 134, 30.4.2004, p. 114

Art 26 335

Directive 2004/48/EC Enforcement of IP rights

Art 14 . .250

Directive 2005/29/EC Unfair commercial practices [2005] OJ L $149 / 22$ $24,164,254$

Art 2(f) 161

Art 2(g) 161

Art 6(2)(b). $68,90,117,161$

Art 10 161

Directive 2005/36/EC Recognition of professional qualifications [2005] OJ L255/22 (as amended) 151

Directive 2006/123/EC Services in the internal market [2006] OJ L376

rec 114

Art 3/7.

Art 3/9

Art 10 150
Art. 37/1 150

Directive 2008/48/EC Consumer Credit, OJ L 133, 22.5.2008, p. 6 356

Directive 2009/72/EC Common rules for the internal market in electricity (repealing Directive 2003/54/EC) OJ L 211, 14.8.2009, pp. 55-93 ……........................ 264

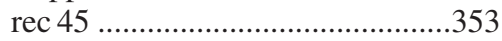

Art 6(4) ......................................264

Art 21(2)............................264, 277

Art 21(3) ....................................272

Art 21(6)...........................277, 278

Art 21(10) ..................................272

Art 21(11) ..................................272

Directive 2009/73/EC Common rules for the internal market in natural gas (repealing Directive 2003/55/ EC) OJ L 211, 14.8.2009, pp. 94-136 ............................ 264

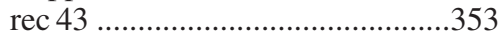

Art 7(4) ...........................................264

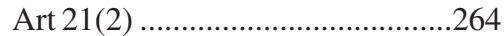

Annex 1, s 1 ....................................353

Directive 2009/138/EC Solvency II, OJ L 335, 17.12.2009, pp. $1-155$ 272

Art 46 .... 264,272

Directive 2010/13/EU Audiovisual media services [2010] OJ L95/1

Art. 9/2 .150

Directive 2011/83/EU Consumer rights 4

Directive 2013/11/EU Alternative dispute resolution for consumer disputes (amending Regulation (EC) No 2006/2004 and Directive 2009/22/EC (Consumer ADR)), OJ L 165, 18.6.2013, p. 63 348-349

Directive 2014/17/EU Mortgage Credit, OJ L 60, 28.2.2014, p. 34

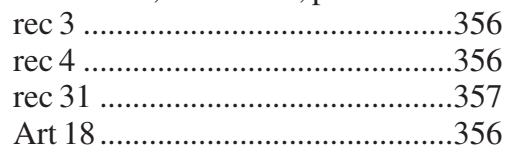


Directive 2014/24/EU Public procurement (repealing Directive 2004/18/EC), OJ L 94, 28.3.2014, p. 65 334

Directive 2014/65/EU Markets in financial instruments (amending Directive 2002/92/EC and Directive 2011/61/EU) OJ L 173 , 12.6.2014, pp. 349-496

Art 31

Art 54

Directive 2014/104/EU Actions for damages under national law for infringements of the competition law provisions of the Member States and of the European Union, OJ L 349, 5.12.2014, p. 1

Art 11 327

Directive (EU) 2015/849 Money laundering or terrorist financing (amending Regulation (EU) No 648/2012 and repealing Directive 2005/60/EC and Directive 2006/ 70/EC) OJ L 141, 5.6.2015, pp. 73-117 263

Art 8(4) 264

European Convention on Human Rights 152

Guidelines on State aid to airports and airlines (2014), 2014/ C 99/03 268 para 30 267

Regulation (EC) No 1/2003 Competition Rules, OJ L 1, 4.1.2003, pp 1-25 261,262 Art 9

Art 27(4) 268

Regulation (EC) No 139/2004

Mergers, OJ L 24, 29.1.2004, pp 1-22 261,266

rec 30 266

Art 6.2

Regulation (EC) No 864/2007 Law applicable to non-contractual obligations (Rome II) OJ L 199, 31.7.2007, p. 40
Art 26 342

Regulation (EC) No 593/2008 Law applicable to contractual obligations (Rome I) OJ L 177, 4.7.2008, p. 6

Art 6(1) 343

Art 6(2) 343

Art 8(1) 343

Art 8(2) 343

Art 21 342

Regulation (EC) No 1060/2009 Credit rating agencies, OJ L 302, 17.11.2009, pp. 1-31 263

Annex I .278

Regulation (EU) No 330/2010 Application of Article 101(3) of the TFEU to categories of vertical agreements and concerted practices, OJ L 102, 23.4.2010, p. 1

Art 2(2) 339

Art 2(4) 339

Art 3 339

Art 4 339

Regulation (EU) 305/2011 Marketing of Construction Products (repealing Directive 89/106/EEC) [2011] OJ L88/5 151,159

Regulation (EU) No 1025/2012 European standardisation [2012] OJ L316/12 158,355

Regulation (EU) No 806/2014 Single Resolution Mechanism and a Single Resolution Fund (amending Regulation (EU) No 1093/2010), OJ L 225, 30.7.2014, pp. $1-90$

Art 19(3) 268

Regulation (EU) 2016/679 General Data Protection (GDPR) (repealing Directive 95/46/EC) [2016] OJ L 119/1 ... 44, 260, 269, 271

rec 97 .279

Section 4............................262, 263

Art 9 270

Art 10 270 
Art 37

Art 37(1)

Art 37(5)

..270

Art 38

$.263,275$

Art 38(3)

263

Art 38(5)

Art 38(6)

279

Art 39

Art 99

279

279

263, 274

Treaty on European Union (TEU)

Art 19 271

Treaty on the Functioning of the

European Union

153

Art 101 $148,154,162,163,339$,

Art 101/1 $340,341,361,362$

Art 101/2 154,361

Art 102 161,164

Art 106 $148,154,155$ 155

\section{National Legislation}

\section{Australia}

Franchising Code of Conduct 209

\section{Germany}

Civil Code

Federal Data Protection Act 2003, as amended

$\S 4 \mathrm{f}(1)$

$\S 4 \mathrm{f}(2)$

$\S 4 \mathrm{f}(3)$

$\S 43(3)$

Penal Code

s 13

\section{Italy}

Legislative Decree no 231 of 8 June 2001 265

\section{Poland}

Act on the Protection of Personal Data

$$
1997
$$

Art 36a(5)

275

Art 36a(7)

Art 36a(8)....................................279

Art 36b

Criminal Code

Art $296 \S 1$

\section{Netherlands}

Civil Code (BW)

Art. 6:213 lid 2 ..............................242

Art 6:253(4) ....................................74

Art 6: 265 ......................................242

Art 6:279 ....................................242

Art 7A: 1670 ...................................250

Copyright Act

Art 11 .............................................12

Financial Supervision Act

art 4:34.

Judiciary Organization Act (RO) ...... 78

Art 79

$.78,79$

Wet kwaliteit, klachten en geschillen zorg (Staatsblad 2015/407, in force as of 1 January 2016) Art 3

\section{Spain}

Criminal Code 265

\section{United Kingdom}

Contracts (Rights of Third Parties) Act 1999 $173,178,191,193$

Sale of Goods Act 1979

S 14

Statute of Frauds 1677

Unfair Terms in Consumer Contracts Regulations 1999, SI 1999/2083

Sch 2, para 1(i)

\section{United States of America}

Sarbanes-Oxley Act

s 402 .

s 406 .

Restatement (Second) of Contracts

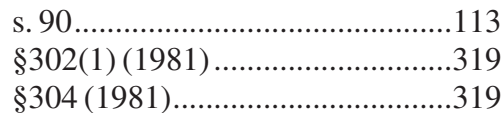




\section{International, National and Corporate Codes and Instruments}

Advertising Code (Netherlands) ..... 43,

69

British Franchise Association, Code of Ethics 209

Charter of core principles of the European legal profession 152

Chiquita, International Banana

Purchase Agreement .... 296, 300, 303, 305

Code of Conduct for Energy Suppliers

(Netherlands) 43

Code of Conduct for European lawyers [2008] CCBE $138,143,144$,

Art 1.3.1 138,144

Art 3.1.3 152

Code of Conduct for Pharmaceutical

Advertising (Netherlands) ....... 87

Code on Consumer Credit

(Netherlands) 69

Common Frame of Reference (CFR) 3

Draft Common Frame of Reference (DCFR) $3,57,162,208$

Art I.-1:103-1:104 . .163

Art II.-1:101 .63

Art II.-1:101(1) .52

Art II.-1:104 163

Art II.-4:102 .64

Arts II.-7:301-302 ..71

Art II.-9:301 .317

Art II.-9:301(1) 62

Arts II.-9:403-408 .88

Draft United Nations Code of Conduct on Transnational Corporations, UN Doc. E/C.10/1982/6, 5 June 1982 (revised UN Doc. E/1983/ 17/Rev.1) 103

EPI Code of Conduct 149

ESTI Statutes

Art 8 147
European Code of Ethics for Franchising ..... 45, 73, 76-78, 81, 82

Art 4.3. .76

European Codes of Conduct for Direct

Selling (2011), European Direct

Selling Association (Seldia) ..... 43

EU Framework for Online Behavioural

Advertising, IAB Europe ......... 43

Fair Labor Association, FLA Workplace Code of Conduct and Compliance Benchmarks (2011) ....... 145, 284, 289

FIFA Players' Agents Regulations (2008) (Fédération Internationale de Football Association) ....... 155, 166

Code of Professional Conduct (Annexe 1) 142

Standard agency contract (Annexe 3)

Forest Stewardship Council, 'Principles and Criteria for Forest Stewardship, Version 5.2 (2015) 284

GLOBALGAP Integrated Farm

Assurance module for Fruits and

Vegetables 310

Intellectual Property Rights (IPR)

Policy, ETSI (European

Telecommunications Standards Institute) 146,158

Art 6.1 147

Art 14 147

Model Trustee Mandate ........ 273, 275, 278,280

Netherlands Home shopping association, Rules 69

New York Stock Exchange Listed Company Manual

s. 303A.10. 101

OECD Guidelines for Multinational Enterprises 1976 (last update 2011) 103,114

Principles of European Contract Law (PECL) 57,208 art 2:102 64 art $4: 110$ . .88 
art 6:110 .317

art 6:110(1) .62

arts 15:101-102 71

Rules concerning trade on the options exchange (RHO)

(Netherlands)

SMS Service Provision Code of

Conduct (Netherlands) 73,79 $73,74-75,80$

Art 1 . .74

Art 2(1) .62

UN Guiding Principles on Business and Human Rights 2011 (A/HRC/17/ 31) endorsed by the UN Human Rights Council on 6 July 2011 (A/HRC/RES/ 17/4) ....... 103, 105

UNIDROIT Principles of International Commercial Contracts 208
Article 5.2.1 317

Unilever, General Terms and Conditions for the Purchase of Products and Services (Version 1 November 2015) ... 297, 298, 299, 302, 306, 308

Unilever, Responsible Sourcing Policy ...... 298, 299, 306, 307, 308

Unilever, Unilever's Supplier Qualification System .... 298, 299, 302,304

Volkswagen Group, code of conduct 107

Wal-Mart Code of conduct (Standards for Suppliers) 318

World Anti-Doping Code (WADC) 141,142

Art 23 142 